\title{
Low Temperature Magnetoelasticity and Internal Friction of an Amorphous Reentrant Spin Glass
}

\author{
E. Gaganidze, A. Setzer and P. Esquinazi \\ Dept. Superconductivity and Magnetism, University of Leipzig, Linnéstrasse 5, 04103 Leipzig, \\ Germany
}

\begin{abstract}
We have studied the magnetomechanical behavior of the amorphous metal METGLAS 2826A $\left(\mathrm{Fe}_{32} \mathrm{Ni}_{36} \mathrm{Cr}_{14} \mathrm{P}_{12} \mathrm{~B}_{6}\right)$ in the as-quenched and annealed states at temperatures $50 \mathrm{mK}<\mathrm{T}<10 \mathrm{~K}$, magnetic fields $\mathrm{B}<3 \mathrm{~T}$ and at frequencies $<3 \mathrm{kHz}$. The alloy is ferromagnetic below $240 \mathrm{~K}$ and shows a reentrant spin glass transition at $\mathrm{T}_{\mathrm{f}}$ $=1.2 \mathrm{~K}$. The acoustic properties in the as-quenched and in the early stage of annealing states show strong anomalies at the spin glass transition. These anomalies vanish for a field of $0.07 \mathrm{~T}$ applied parallel to the main surface of the sample. Due to demagnetization effects the field dependence of magnetoelastic properties at $T_{f}$ depends strongly on the angle between magnetic field and main surface of the sample. We show that the "Giant $\Delta \mathrm{E}$ effect" observed in this sample is a consequence of the macroscopic magnetization of the sample. The sample in the amorphous and partially crystallized states shows no glasslike behavior in the acoustic properties which is usually observed in amorphous and polycrystalline metals.
\end{abstract}

\section{INTRODUCTION}

The aim of our work is threefold: (a) It is well known that magnetic interactions have an influence not only on the magnetic properties but also on the elastic behavior of solids. The phenomenon of magnetoelasticity results from the interaction between the magnetic entities (spins, clusters, domains...) and elastic waves. The ferromagnetic (FM) to spin glass (SG) transition attracted considerable attention the last 20 years (see for example [1]). However the acoustic properties of metallic spin glasses in the $\mathrm{kHz}$ range were not thoroughly studied. Investigation of acoustic properties of metallic spin glasses near the spin freezing temperature $\mathrm{T}_{\mathrm{f}}$ can provide useful information about spin-phonon coupling and is a tool to study spin freezing phenomena in addition to the classical methods as magnetization, ac susceptibility, etc.

(b) In spite of a diversity of investigations of the magnetomechanical behavior of amorphous ferromagnetic ribbons the phenomena called "Giant $\Delta \mathrm{E}$ effect" is not completely understood $[2,3]$. Investigation of acoustic properties under applied magnetic field in parallel and perpendicular geometries is necessary in order to distinguish between the magnetoelasticity itself and variation of resonance frequency due to macroscopic magnetization.

(c) The study of the acoustic properties after heat treatment of the samples provides new information about the structural relaxation and structural or magnetic phase transitions of the system. The sensitivity of the method is high and its information is complementary to standard methods as DTA and XRD.

\section{EXPERIMENTAL DETAILS}

The sample is a commercially available amorphous ribbon METGLAS $2826 \mathrm{~A} \quad\left(\mathrm{Fe}_{32} \mathrm{Ni}_{36} \mathrm{Cr}_{14} \mathrm{P}_{12} \mathrm{~B}_{6}\right)$ produced by Allied Chemical Corp., USA. The alloy is ferromagnetic below $\mathrm{T}_{C}=240 \mathrm{~K}$ and undergoes a 
reentrant transition to a spin glass state at $T_{f}=1.2 \mathrm{~K}$ (defined as the temperature at which a maximum in the sound attenuation occurs or the temperature at which the magnetic susceptibility decreases). For the acoustic measurements we have used the vibrating reed technique. The samples had a length of (37) $\mathrm{mm}, 1.5 \mathrm{~mm}$ width and $50 \mu \mathrm{m}$ thickness. Typical frequencies of the reeds were from $600 \mathrm{~Hz}$ to $3 \mathrm{kHz}$. To study the magnetomechanical behavior we investigated the field dependence of the resonance frequency and damping in parallel (dc field applied parallel to the vibration axis or width of the reed) and perpendicular (to the main surface of the reed) geometries. To exclude variation of the elastic constants due to strain-induced domain movement we also performed measurements in a strain free state by gluing a piece of METGLAS to a host reed made from silicon single crystal. Acoustic investigations were performed in a dilution refrigerator allowing measurements from $50 \mathrm{mK}$. Magnetic field was provided

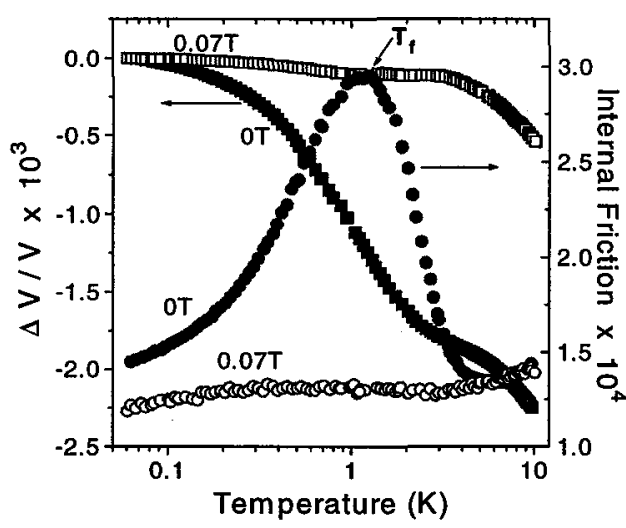

Figure 1. Relative change of the sound velocity (squares) and the corresponding internal friction (circles) as a function of temperature in zero and $0.07 \mathrm{~T}$ applied fields. Frequency of the measurement was $2.7 \mathrm{kHz}$. by a $8 \mathrm{~T}$ superconducting magnet. Dc magnetization, DTA and XRD technique were used to characterize the samples in the as-quenched and annealed states.

\section{EXPERIMENTAL RESULTS AND DISCUSSION}

\subsection{Acoustic Properties at Spin Freezing Temperature}

Fig. 1 shows the acoustic properties of the as-quenched sample near the freezing temperature $T_{f}$ in zero and 0.07T applied fields. In zero field strong anomalies are observed around the freezing temperature: a relatively broad internal friction peak at $\sim 1.2 \mathrm{~K}$ accompanied by an increase of the Young modulus sound velocity on cooling. The sound velocity exhibits an inflection point at the temperature where attenuation shows a maximum. At the temperature $T_{f}$ long range ferromagnetic order, characteristic for the FM state at higher temperatures, is destroyed and transformed into a cluster glass state. The freezing temperature deduced this way is in good agreement with that one obtained from ac susceptibility measurements [4]. Acoustic measurements at two different frequencies show that this $T_{f}$ is only a dynamic manifestation of a possible "phase transition", i.e. we observed $220 \mathrm{mK}$ shift in $T_{f}$ by changing the frequency from $600 \mathrm{~Hz}$ to $2.7 \mathrm{kHz}$. The broadness of the internal friction peak suggests the existence of a relaxation time spectrum rather than a single relaxation time [5]. The origin of the spectrum lies in the amorphousness and magnetic disorder of our system, a mixture of different entities (magnetic domains, clusters, single spins...).

A magnetic field has a large influence on the acoustic properties (as is the case for the ac magnetic susceptibility in canonical spin glass systems [1] ). A field of $0.035 \mathrm{~T}$ applied in parallel configuration reduces the internal friction at $T_{f}$ by factor of two. At $0.07 \mathrm{~T}$ the maximum in the internal friction completely smears out, see Fig.1. One has to mention that $0.07 \mathrm{~T}$ is the field at which magnetization saturates. This means that spin-glass transition disappears when the applied field is strong enough to align small clusters and single spins along the field direction. In the case of perpendicular geometry to suppress completely the spin glass transition and because of demagnetization effects we need to apply a field of $1 \mathrm{~T}$ instead of $0.07 \mathrm{~T}$.

We note that in the as-quenched state and at an applied field large enough to suppress the spin glass transition, no signs of glass-like anomalies are observed in the acoustic properties. The same result is obtained for the annealed and partially crystallized states. The low temperature acoustic properties of amorphous materials as well as polycrystalline metals show characteristic anomalies due to the interaction of phonons with tunneling two-level systems (TS) [6]. It can be speculated that the magnetism may have some influence on the phonon-TS interaction. However, recent results obtained on the polycrystalline ferromagnetic FeNiMn indicate that this is not always the case [5]. 


\subsection{Variation of the Frequency of the Vibrating Magnetic Reed under a Magnetic Field}

In Fig. 2.a and 2.b we present the normalized change of the resonance frequency of the reed as a function of the applied magnetic field in perpendicular and parallel geometries, respectively.
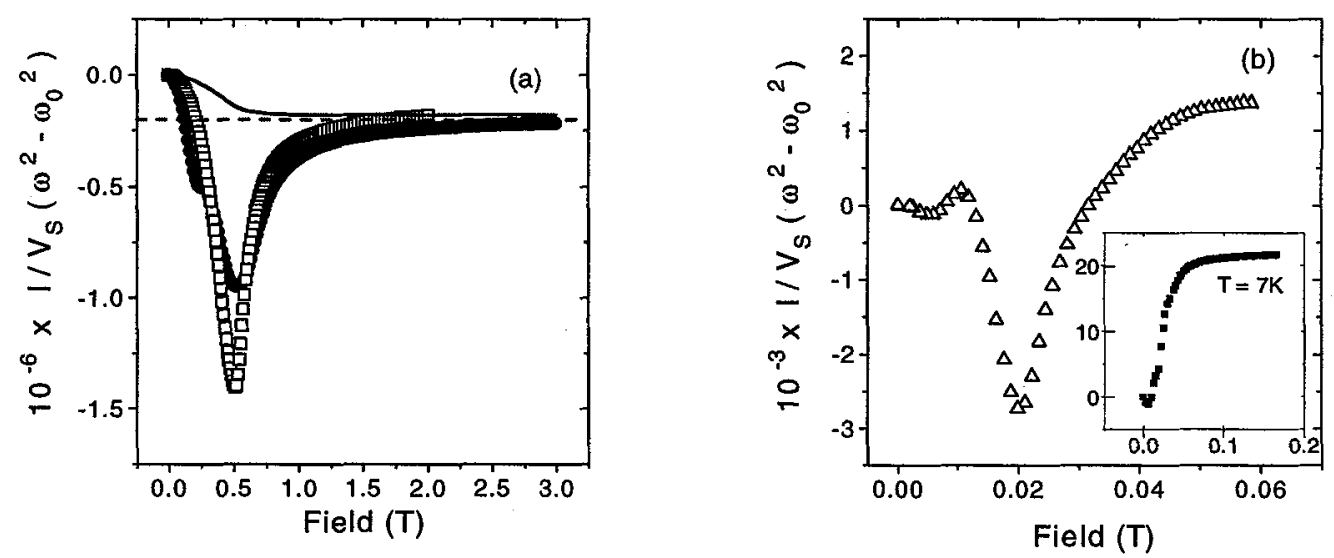

Figure 2: Normalized change of the resonance frequency square as a function of field in perpendicular (a) and parallel geometry (b) at a temperature $\mathrm{T}=0.2 \mathrm{~K}$. The points $(\square)$ were obtained from a sample glued on a silicon single crystal. Solid line represents calculated resonance frequency change due to the anisotropy energy. Dashed line represents the expected resonance frequency change at magnetically saturated case taking into account the measured anisotropy constant. "I" denotes the inertia moment and " $V_{s}$ " the sample volume. Inset represents measurements at $T=7 \mathrm{~K}$.

Measurements were done in the $S G$ phase at $T=0.2 \mathrm{~K}$ as well as at $T=7 \mathrm{~K}>T_{r}$. Because of negligible quantitative difference between SG and FM state in perpendicular geometry only SG state results are shown, while in parallel geometry due to qualitatively different behavior we also present the result in FM state. At first glance the two curves look quite similar: well-defined minima in the resonance frequency are observed. But the origin of these minima is different. In order to understand the phenomena we have to take into account the structure of magnetic domains that are mostly $180^{\circ}$ domains with magnetic moments oriented in the ribbon plane. The anisotropy constant deduced from the magnetization measurements is $\sim 1 \times 10^{5} \mathrm{~J} / \mathrm{m}^{3}$. The corresponding anisotropy $1 / 2 \mu_{0} \mathbf{M}^{2} \cos ^{2}(\Theta)$ term should be taken in the free energy in addition to the magnetic energy $-\operatorname{MBcos}(\Theta)$ of the sample $(\Theta$ is measured from the axis of hard direction i.e. normal to the ribbon plane). The condition of the energy minimum (i.e. condition of the balance between the anisotropy energy torque $\tau_{A}$ and the torque exerted by the external field $\tau_{\mathrm{M}}$ ) indicates that when the magnetic field is applied parallel to the hard axis the in plane magnetization is zero.

In the case of perpendicular geometry the decrease of the resonance frequency is not due to the "softening of the elastic constant" as it is often considered in the literature (see for example [2,3]), but due to the torque (originating in the anisotropy energy) acting on the magnetic reed. The change of the square of the frequency $\omega$ is the first derivative of the torque and thus the second derivative of the anisotropy energy $\mathbf{F}_{\mathrm{A}}$ (assuming the energy minimum condition) with respect to an angle $\Theta$ between applied magnetic field and the magnetization vector: $\omega^{2}-\omega_{0}{ }^{2}=\left(V_{s} / I\right) \partial^{2} F_{A} / \partial \Theta^{2}$, where $\omega_{0}$ is the frequency corresponding to the demagnetized case, $\mathbf{V}_{\mathbf{s}}$ is the volume of the sample and $\mathbf{I}$ the inertia moment. Corresponding resonance frequency change is presented in Fig. 2 (a) by the solid line. In magnetically saturated case (B $>2 \mathrm{~T}$ ) nice agreement is obtained. The fact that the observed frequency change deviates from the theoretical one in the non saturated region indicates the unbalance between $\tau_{\mathrm{A}}$ and $\tau_{\mathrm{m}}$ due to pinning of magnetic domains. The dashed line in Fig. 2 (a) renresents the exnester 
resonance frequency change at magnetically saturated case taking into account the anisotropy constant deduced from the magnetization measurement. The observed agreement indicates that the vibrating reed measurement can be successfully used for obtaining anisotropy constant.

In the case of parallel geometry the observed minimum (Fig. 2 (b)) can be related to the softening of the elastic constant which results from the stress induced domain movements. Note that the absolute value of the variation of the squared frequency in this case is about factor 400 lower. This variation can be understood [7] according the theory developed in Ref. [3].

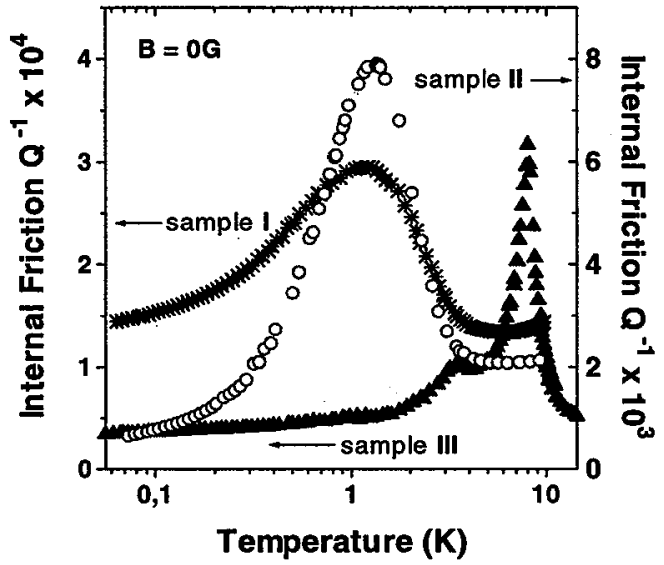

Figure 3. Internal friction for differently heat treated samples (see text) versus temperature in zero applied field. Note that for sample II right axis is valid.
In order to confirm that in the case of perpendicular geometry the frequency change is due to the torque acting on the ferromagnetic reed we performed measurement in the stress-free case: a small piece of METGLAS was glued to the host reed made from single silicon crystal. The results are shown in Fig. 2(a). Excellent agreement is obtained.

\subsection{Effect of the Heat Treatment}

The kinetics of crystallization of Metglas 2826A was thoroughly investigated in Ref. [8]. It was found out that the Metglas $2826 \mathrm{~A}$ displays a two-stage crystallization involving formation of metastable MSI and MSII crystalline phases [8]. Fig. 3 shows the internal friction of the as-quenched and heat treated samples at $1 \mathrm{~h}, 350^{\circ} \mathrm{C}$ and $1 \mathrm{~h}, 410^{\circ} \mathrm{C}$ called sample I, II and III respectively. Thirty times larger internal friction peak for sample II is observed. This increase might be related to an increase of the number of freezing spins (clusters) or an increase of spinphonon coupling constant; according to DSC thermograms the formation of MSI phase in sample II already started, in agreement with a diffraction peak developed at $2 \Theta=44^{\circ}$. Formation of MSII crystalline phase in sample III (according to DSC as well as XRD measurements formation of MSII phase begins already after $1 \mathrm{~h}, 370^{\circ} \mathrm{C}$ heat treatment) qualitatively changes the acoustic properties of the system, see Fig. 3. Reentrant behavior as well as the ferromagnetism disappears (the latter follows from the magnetization measurement versus temperature). A sharp cusp developed in the internal friction at $8 \mathrm{~K}$ may be related to a structural and magnetic phase transition observed also in magnetization measurements. More details on the influence of the crystallization process on the acoustic and magnetic properties of the sample will be published elsewhere.

\section{ACKNOWLEDGMENTS}

This work is supported by the Deutsche Forschungsgemeinschaft under Grant Es 86/2-2.

\section{References}

[1] Mydosh J.A., "Spin glasses: an experimental introduction", Taylor\&Francis, London 1993.

[2] Berry B.S. and Pritchet W.C., J.Appl.Phys. 47 (1976) 3295-3301.

[3] V. Haslar et al., J.M.M.M. 153 (1996) 63-74; J. Barandiaran et al., J.M.M.M. 40-144 (1995)273-274.

[4] Stosch S., Diplomarbeit, 26.04.1991, Universität Bayreuth, EPV.

[5] E.Gaganidze and P.Esquinazi, Int. Conf. on Low Temp. Phys. LT21 (to be published).

[6] P.Esquinazi, R.König and F.Pobell, Z.Phys.B 87 (1992) 305.

[7] E.Gaganidze and P.Esquinazi, (to be published).

[8] M. Von Heimendahl and G. Maussner, J. Mater. Sci. 14 (1979) 1238. 